\title{
Comment on case series of COVID-19 in patients with myasthenia gravis: a single institution experience by Županić et al.
}

\author{
Giuliana Galassi $^{1}$ D $\cdot$ Alessandro Marchioni $^{2}$
}

Received: 6 June 2021 / Accepted: 2 July 2021 / Published online: 4 October 2021

(c) Belgian Neurological Society 2021

Keywords COVID-19 $\cdot$ Myasthenic crisis $\cdot$ Myasthenia gravis $\cdot$ Noninvasive ventilation $\cdot$ Mechanical ventilation

Županić et al. [1] described eight patients, six males and two women, with pre-existing diagnosis of myasthenia gravis (MG) and COVID-19 infection. In four cases, acetylcholine receptor $(\mathrm{AChR})$ antibodies were detected, while two were seronegative, and the remaining untested. Interestingly, at the time of the SARS-CoV-2 infection, three cases were asymptomatic, whereas one was in stage 1 , two in stage $2 \mathrm{~b}$, and one in stage $3 \mathrm{~b}$ according to Myastenia Gravis Foundation of America score (MGFA) [2]. The authors [1] stated that four patients (case 1, 2, 4, 7) showed clear-cut worsening symptoms of MG on admission, but apparently only two of those (case 4 and 7) exhibited a deepening in MGFA severity; in one case (8) who was admitted because of respiratory failure, after fever, cough and dyspnea, the neurological assessment was inconclusive. Pneumonia was reported in six; among those, four received remdesivir added to dexamethasone in one and dexamethasone alone in another. Out of the eight, non-invasive oxygen therapy was applied in two: case 3, asymptomatic during hospital stay and 7 who was classified as $2 \mathrm{~b}$ in MGFA score. In such patients, we would considered the need of respiratory support due the SARS-CoV-2 pneumonia. Interestingly, only one patient (case 4) showed a myasthenic crisis (MC) which needed mechanical ventilation (MV). Death occurred only in one patient, who died after 11 days of intensive-care unit (ICU), while the remaining had fair outcome. Six patients of Županić et al. [1] received intravenous immunoglobulins (IVIG) at usual dose, eventually added to their baseline

Giuliana Galassi

giulianagalassi@alice.it

1 Department of Biomedical, Metabolic, and Neural Sciences, University Hospital, Via P Giardini, 1350, 41010 Modena, Italy

2 Respiratory Diseases Unit, University Hospital, Via P Giardini, 1350, 41010 Modena, Italy therapy. The description of this case series is interesting and deserves a brief comment.

First, while for other neurological manifestations of central nervous system, it can be easy to establish a relation with COVID-19, in case of neuromuscular disorders, clinicians must be cautious in attributing the symptoms to worsening of the neurological status rather than to the COVID-19- itself $[3,4]$. About $20 \%$ of patients with SARS-COV-2 exhibit respiratory weakness and failure, due to viral infection [5]. The development of selective respiratory muscles weakness could be a feature of COVID-19-related MG exacerbation, and may be triggered by increased inspiratory effort due to respiratory drive activation in the course of concurrent pneumonia [4]. In this scenario, early non-invasive pressure support could prevent fatigue and potentially avoid the need for MV in patients without significant alterations in gas exchange. Indeed, endotracheal intubation and MV, despite being the gold standard of treatment for acute respiratory failure due to $\mathrm{MC}$, are associated with complications, including pneumonia and atelectasis, as well as prolonged ICU stays and difficult weaning [6]. Furthermore, some retrospective studies show that about $40 \%$ or more of MC patients undergoing MV fail weaning trial and require tracheostomy [6].

Second, Županić et al. [1] do not provide details about severity of the concurrent pneumonia and radiological results; these data could clarify the interplay between $\mathrm{MG}$ and COVID-19 infection. Also, a functional respiratory muscle assessment might help to identify the development of weakness and the timing of initiation of non-invasive ventilation. However, given the fluctuating nature of muscle fatigue typical of MG, the respiratory functional assessment may also not be able to predict the need of ventilatory support.

Third, we think that the decisions on management of patients with generalized MG and SARS-CoV-2 infection 
should be taken thorough careful neurological surveillance. A cautious administration of IVIG is recommended in patients with concomitant SARS-CoV-2 infection, because widespread thrombosis has been reported in critically ill COVID-19 patients, as previous authors pointed out $[1,5$, 7]. According to the literature, the IVIG treatment or plasma exchanges should be introduced in MG in case of clear-cut acute exacerbations of respiratory and neurological symptoms and in conditions defined as MC [6, 7]. The rational of the use of IVIG should be a short-term treatment in patients with life-threatening signs or when a rapid clinical response is needed or when other therapies are insufficiently effective $[6,7]$. Finally, the Brazilian series [8] with $30 \%$ rate of death in MG hospitalized with COVID-19 describes patients with features falling into the definition of $\mathrm{MC}$, as $87 \%$ were admitted to ICU and $73 \%$ needed MV.

\section{Declarations}

Conflict of interest The authors declare that they have no conflict of interest.

Ethical approval This article does not contain any studies with human participants or animals performed by any of the authors.

Informed consent Informed consent was obtained from all individual participants included in the study.

\section{References}

1. Županić S, Perić Šitum M, Majdak M et al (2021) Case series of COVID-19 in patients with myasthenia gravis: a single institution experience. Acta Neurol Belg 1:1-6. https://doi.org/10.1007/ s13760-021-01662-w

2. Jaretzki A, Barohn RJ, Ernstoff RM et al (2000) Myasthenia gravis: recommendations for clinical research standards. Task Force of the Medical Scientific Advisory Board of the Myasthenia Gravis Foundation of America. Neurology 55:16-23. https:// doi.org/10.1212/wnl.55.1.16

3. Anand P, Slama MCC, Kaku M et al (2020) COVID-19 in patients with myasthenia gravis. Muscle Nerve 62:254-258. https://doi. org/10.1002/mus.26918

4. Galassi G, Marchioni A (2021) Myasthenia gravis at the crossroad of COVID-19: focus on immunological and respiratory interplay. Acta Neurol Belg 121(3):633-642. https://doi.org/10.1007/ s13760-021-01612-6

5. Wu C, Chen X, Cai Y et al (2020) Risk factors associated with acute respiratory distress syndrome and death in patients with coronavirus disease 2019 pneumonia in Wuhan, China. JAMA Intern Med 180:934-943. https://doi.org/10.1001/jamainternmed. 2020.0994

6. Neumann B, Angstwurm K, Mergenthaler P et al (2020) Myasthenic crisis demanding mechanical ventilation: a multicenter analysis of 250 cases. Neurology 94(3):e299-e313. https://doi. org/10.1212/WNL.0000000000008688

7. Delly F, Syed MJ, Lisak RP, Zutshi D (2020) Myasthenic crisis in COVID-19. J Neurol Sci 15(414):116888. https://doi.org/10. 1016/j.jns.2020.116888

8. Camelo-Filho AE, Silva AMS, Estephan EP et al (2020) Myasthenia gravis and COVID-19: clinical characteristics and outcomes. Front Neurol 11:1053. https://doi.org/10.3389/fneur.2020.01053 (eCollection)

Publisher's Note Springer Nature remains neutral with regard to jurisdictional claims in published maps and institutional affiliations. 